\title{
Pericardiectomy for Constrictive Pericarditis after Mediastinal Irradiation - Need for Functional Assessment
}

\author{
Siddharth Pahwa ${ }^{1}$ \\ ${ }^{1}$ University of Louisville Division of Thoracic and Cardiovascular Surgery
}

December 29, 2021

\section{Pericardiectomy for Constrictive Pericarditis after Mediastinal Irradiation - Need for Func- tional Assessment}

Siddharth Pahwa, MD ${ }^{1}$

\section{Institutions}

1 - Department of Cardiovascular and Thoracic Surgery, University of Louisville, Louisville, KY

Word count -497

Corresponding author - Siddharth Pahwa, MD

Department of Cardiovascular and Thoracic Surgery

University of Louisville, Louisville, KY

Phone - 502-588-7600

Email - siddharth.pahwa@uoflhealth.org

Surgical pericardiectomy has been established as the standard of care for constrictive pericarditis (CP). ${ }^{1}$ Radiation induced CP presents a unique challenge. ${ }^{2}$ The effects of radiation on other cardiac structures, including the coronary vasculature and cardiac valves, and the associated myocardial fibrosis and dense plane of adhesions makes surgery especially challenging in this subset of patients. Nozohoor, in their recent invited commentary, rightly mentioned the importance of early surgery with prompt referral to tertiary centers with the ability to care for these complex patients. ${ }^{3}$ We have three comments to make on their excellent article.

Firstly, they mentioned that performing concomitant procedures along with pericardiectomy for CP may have an adverse effect on outcomes. ${ }^{3}$ While this may be true for other subsets of CP, Pahwa and colleagues, in their recent study on the outcomes of pericardiectomy in patients with radiation-associated CP, had shown no difference in survival between patients that underwent pericardiectomy with concomitant cardiac procedures and patients that underwent isolated pericardiectomy. ${ }^{4}$ Secondly, Nozohoor pointed out that the improvement in radiation technology and delivery systems over the years have led to sparing of adjacent normal tissue. ${ }^{3}$ Damage to adjacent myocardium and the pan-cardiac involvement in radiation-induced CP has added to the increased complexity and poor outcomes in this population. While Pahwa et al did not show a statistically significant decrease in mortality over time in their study spanning 18 years, their data suggested an improvement in survival over time, that trended towards significance. ${ }^{4}$ This may be attributed to the improved radiation delivery over time as well, as Nozohoor had discussed.

Lastly, although pericardiectomy for radiation-associated CP is associated with poor long-term outcomes in terms of patient survival ${ }^{4}$, it may be worthwhile to look at the functional outcomes of patients suffering 
from the disease. Nozohoor mentioned that the functional class and quality of life may be greatly improved by surgical pericardiectomy in radiation-associated constriction. ${ }^{3}$ We strongly endorse this suggestion, and believe that surgery remains the gold standard in the management of radiation-associated CP. Further studies are needed to look at functional outcomes, hospital re-admissions and quality of life variables in this complex subset of patients, which may further re-affirm the value of pericardiectomy.

\section{References}

1. Gillaspie EA, Stulak JM, Daly RC, et al. A 20-year experience with isolated pericardiectomy: Analysis of indications and outcomes. J Thorac Cardiovasc Surg. 2016;152(2):448-458.

2. Karram T, Rinkevitch D, Markiewicz W. Poor outcome in radiation-induced constrictive pericarditis.Int J Radiat Oncol Biol Phys. 1993;25(2):329-331.

3. Nozohoor S. Invited commentary: Outcomes of pericardiectomy for constrictive pericarditis following mediastinal irradiation. J Card Surg. 2021 Dec;36(12):4643-4644.

4. Pahwa S, Crestanello J, Miranda W, et al. Outcomes of pericardiectomy for constrictive pericarditis following mediastinal irradiation. J Card Surg. 2021 Dec;36(12):4636-4642. 